\title{
Measuring Protein Structure and Stability of Protein-Nanoparticle Systems with Synchrotron Radiation Circular Dichroism
}

\author{
Stefania Laera, ${ }^{\dagger}$ Giacomo Ceccone, ${ }^{\dagger}$ Francois Rossi, ${ }^{\dagger}$ Douglas Gilliland, ${ }^{\dagger}$ Rohanah Hussain, ${ }^{\dagger}$ \\ Giuliano Siligardi, ${ }^{\neq, \S}$ and Luigi Calzolai, ${ }^{*+\uparrow}$ \\ ${ }^{\dagger}$ European Commission, Joint Research Centre, Institute for Health and Consumer Protection I-21027, Ispra (VA), Italy \\ ${ }^{\ddagger}$ Diamond Light Source, Diamond House, Chilton, Didcot, OX11 0DE, U.K. \\ ${ }^{\S}$ School of Biological Sciences, University of Liverpool, L69 7ZX, U.K.
}

Supporting Information

\section{ABSTRACT:}

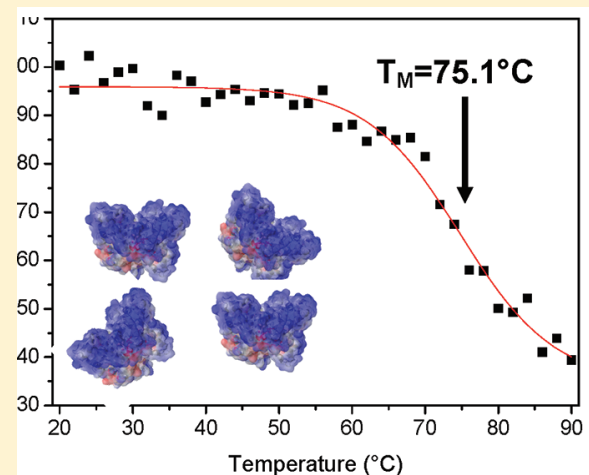

We measure the structural and stability changes of proteins at nanomolar concentration upon interaction with nanoparticles. Using synchrotron radiation circular dichroism (SRCD), we measure a decrease of $6{ }^{\circ} \mathrm{C}$ in the thermal unfolding of human serum albumin upon interaction with silver nanoparticles while this does not happen with gold. The use of SRCD allows measuring critical parameters on protein-nanoparticle interactions, and it will provide experimental data on the relative stability of key biological proteins for nanotoxicology.

KEYWORDS: Protein - nanoparticle interaction, protein structure, protein stability, circular dichroism, synchrotron radiation

U ntil now not much is known about the effect of nanoscale materials on proteins. To understand both the effects of nanoparticles (NPs) in biological systems and to design the next generation NP-based drug delivery systems, it is critical to be able to measure the structure and stability of the protein of interest upon interaction with NPs. This information is very difficult to obtain due to the very nature of the system involving a solid/ liquid interface ${ }^{1}$ and low concentration of the proteins. We measured for the first time the secondary structure of proteins in low nanomolar concentration and in particular the structural and stability changes associated with proteins bound noncovalently to silver and gold nanoparticles close to 1:1 particle ratios using synchrotron radiation circular dichroism (SRCD) technique at the B23 beamline of Diamond Light Source. The use of B23 beamline has allowed detailed structural information on proteinNP complexes that have been elusive until now.

When NPs interact with proteins, they might alter protein conformation, expose new epitopes on the protein surface, or perturb the normal protein function, which could induce unexpected biological reactions and lead to toxicity. ${ }^{2,3}$ The relatively low number of available NPs per unit volume dictates that the concentration of proteins interacting with NPs is low and in the vast majority of cases experiments are run under conditions where the free proteins by far exceed the number of "interacting" proteins. $^{4}$

Circular dichroism (CD) is an excellent and sensitive method for rapidly evaluating the secondary structure, folding and binding properties of proteins, and recently has also been used to detect structural changes of proteins interacting with NPs. ${ }^{5-7}$ The use of synchrotron radiation (SR) to perform CD experiments (SRCD) presents several advantages with respect to the conventional $\mathrm{CD}$ technique. The major advantage is the high flux provided by a SR source with respect to a conventional xenon lamp that allows $C D$ data to be measured both with very low amounts of proteins and in the presence of highly absorbing chemicals such as suspensions of gold and silver nanoparticles. The high photon flux in the far-UV region, small cross size

Received: August 22, 2011

Published: September 20, 2011 

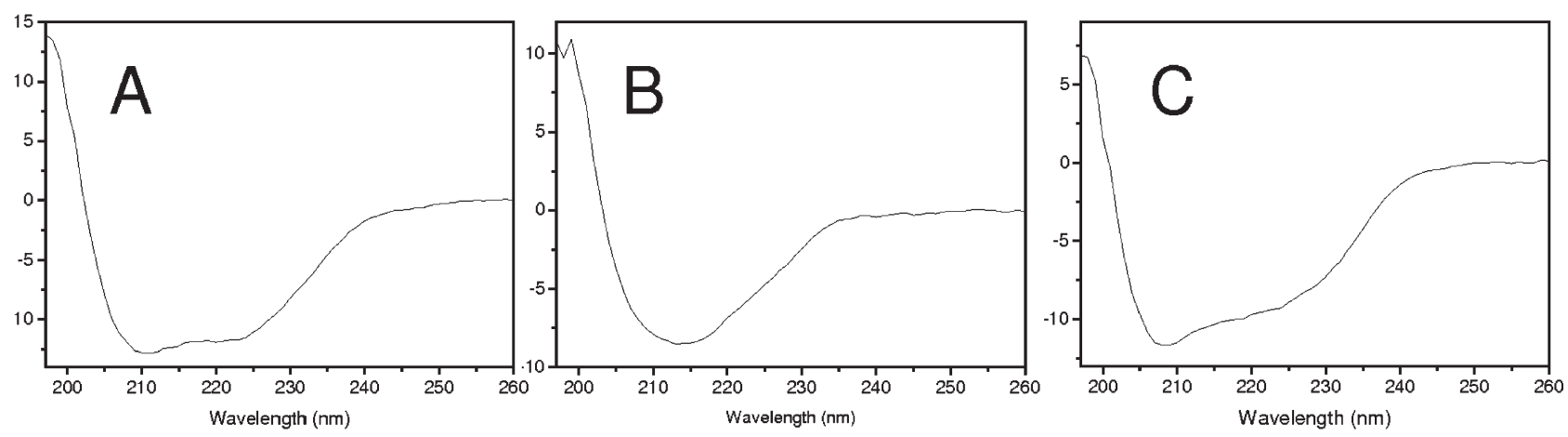

Figure 1. SRCD spectra of proteins measured with a low volume capacity $10 \mathrm{~cm}$ path length cell: (A) HSA $20 \mathrm{nM}$; (B) hTTR $40 \mathrm{nM}$; (C) LYS $100 \mathrm{nM}$.
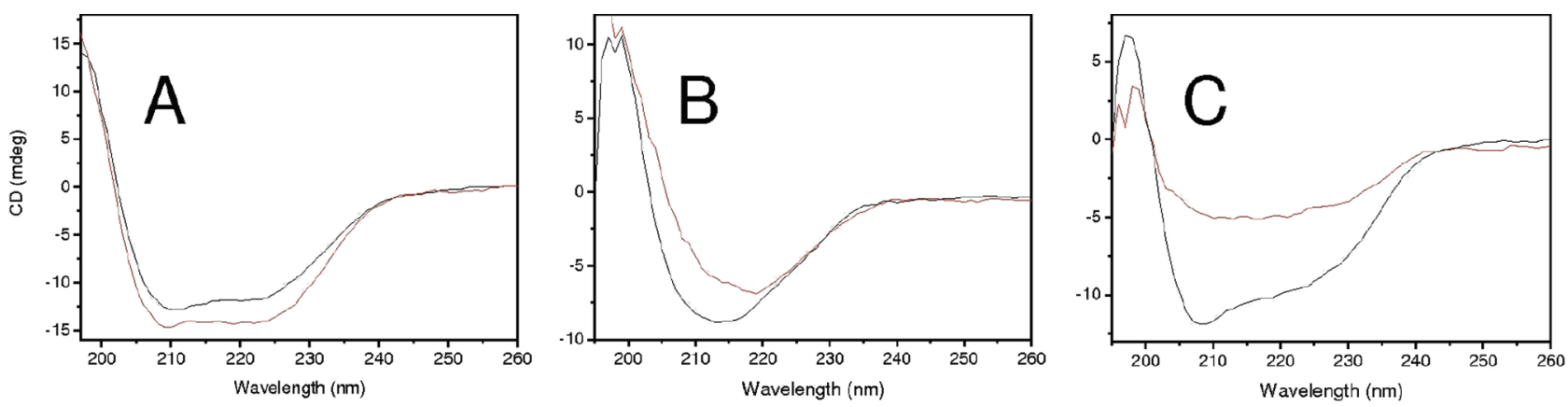

Figure 2. SRCD spectra of protein-AgNP complex (red) and free protein (black) collected with a low volume capacity $10 \mathrm{~cm}$ path length cell: (A) HSA-AgNP 44:1; (B) hTTR-AgNP 88:1; (C) LYS-AgNP 200:1.

section, and highly collimated synchrotron radiation beam from the Diamond B23 CD beamline allows the measurement of CD spectra using a large variety of measurement cells, and in particular long path-length cells with low volume capacity. ${ }^{8,9}$

We show that with SRCD it is possible to measure the secondary structure of selected proteins (human serum albumin, HSA, human transtyrethrin, hTTR, and lysozyme, LYS) in the nanomolar concentration range (corresponding to a few micrograms of protein for each sample) and to detect the structural and stability changes when these proteins form stable, noncovalent, complexes with nanoparticles.

We have chosen proteins of three different structural classes and molecular sizes: a medium-size mainly $\alpha$-protein (HSA), a homotetrameric $\beta$-protein (hTTR), and a small $\alpha-\beta$ protein (LYS). Figure 1 shows the SRCD spectra of the three proteins collected using a quartz cell with a $10 \mathrm{~cm}$ pathway length with $3 \mathrm{~mm}$ diameter and total volume capacity of about $0.8 \mathrm{~mL}$ corresponding to masses of $1.1,1.4$, and $1.1 \mu \mathrm{g}$ for the HSA, TTR, and LYS protein samples, respectively.

These amounts can be reduced by a factor of 2 (see Supporting Information, Figure S1) and probably even further while maintaining data quality by simply collecting more scans. Thus, using just $250 \mathrm{ng}$ of samples and 16 scans, it should be possible to collect CD spectra with the same signal-to-noise ratio as obtained in Figure 1. It is important to note that this cell cannot be used with the traditional bespoke benchtop CD instruments due to their large beam divergence compared to the highly collimated beam generated in the B23 beamline. ${ }^{8}$

The high photon flux of the synchrotron light can cause proteins to denature especially when exposed to radiation below $250 \mathrm{~nm} .{ }^{8,9}$ This effect can be easily monitored by following the changes of the $\mathrm{CD}$ profile of proteins in repeated scan mode. In the case of the long $10 \mathrm{~cm}$ cell, this effect is negligible as can be seen by comparing scan 1 and scan 4 for HSA (see Supporting Information, Figure S2). Similar results have been obtained for the other two proteins.

The CD spectra of the three proteins are consistent with the spectra collected with higher concentrations and shorter path length cells. The use of synchrotron radiation as light source thus allows the detection of the secondary structure of proteins with a few hundred nanograms of sample by examination of the spectral region $195-250 \mathrm{~nm}$ that contains the critical information on the secondary structure of proteins.

The possibility of collecting the CD spectra at nanomolar concentrations has allowed us to measure the CD spectra of HSA, TTR, and LYS in various protein-nanoparticle systems at normality ratios close to $1: 1$.

The CD spectrum of HSA-AgNP (Figure 2A) shows that HSA retains its overall secondary structure upon interaction with $15 \mathrm{~nm}$ AgNP. In the case of hTTR (Figure 2B) the interaction with AgNP causes a clear change in the CD spectrum. An analysis carried out using a basis set of reference CD spectra for $\alpha$-helix, $\beta$-sheet, and irregular structures indicates that in the hTTRAgNP system the protein reduces its limited $\alpha$-helical content and probably increases the irregular one. In the case of LYSAgNP (Figure 2C) the intensity of the CD spectrum decreases significantly compared to the free LYS, while retaining the original shape, thus suggesting that the protein precipitates upon interaction with AgNP. This effect is also confirmed by dynamic light scattering (DLS) measurements which show the formation in solution of some large aggregates after addition of lysozyme to AgNP. 


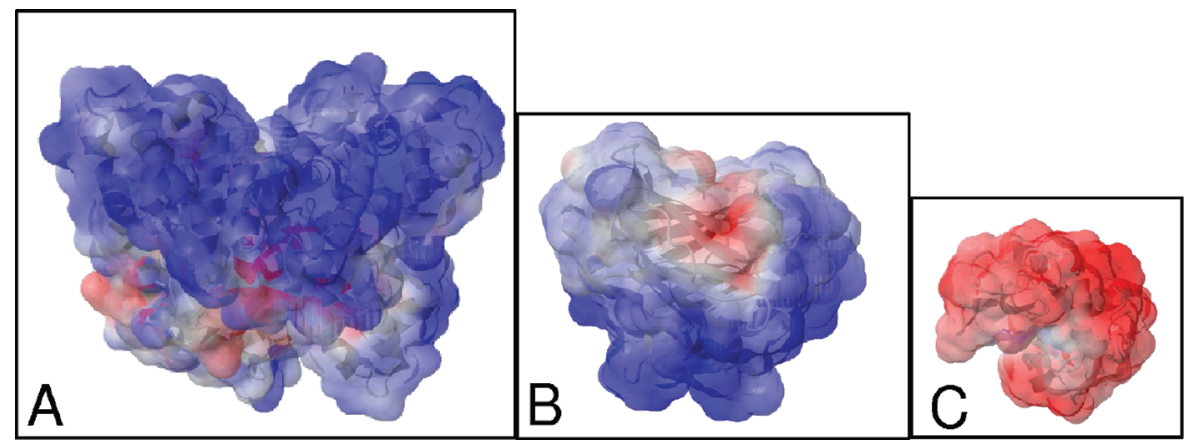

Figure 3. Electrostatic surface potential of (A) HSA, (B) TTR, and (C) LYS, calculated at pH 7.0. The sizes of the pictures are qualitatively related to the relative dimensions of the proteins.
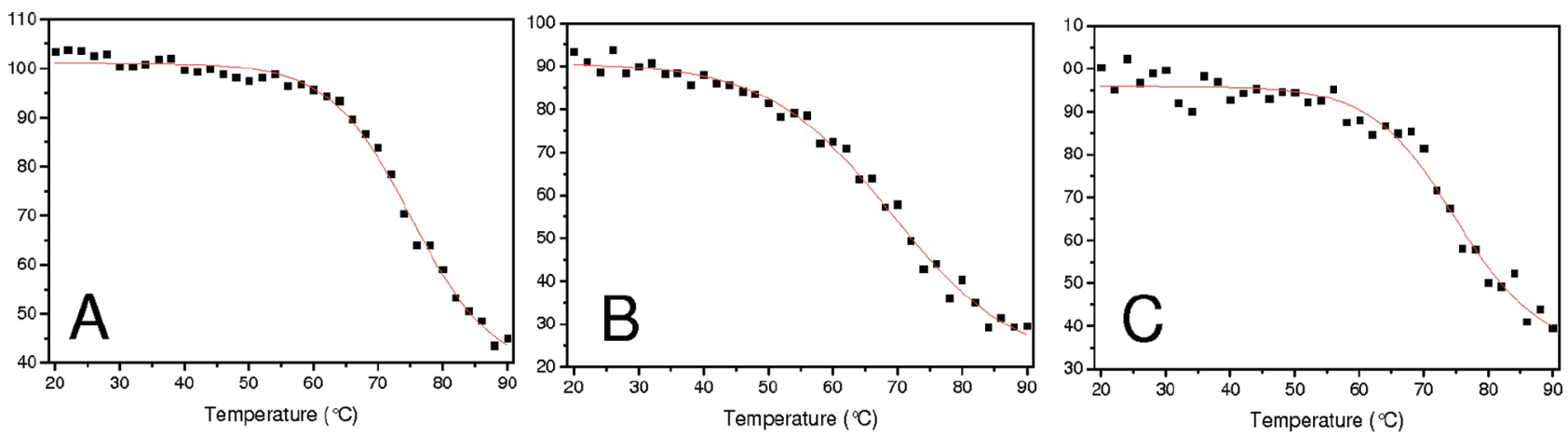

Figure 4. Thermal unfolding of HSA - nanoparticle systems (black squares) fitted with a sigmoidal equation (red line): (A) free HSA; (B) HSA-AgNP 18:1; (C) HSA-AuNP 22:1.

The number ratios of protein molecules to nanoparticles for each of the three systems are about 44:1, 86:1, and 200:1 for the HSA-, TTR - , and LYS-AgNP samples, respectively. On the basis of pure geometrical considerations, it is possible to give an upper limit for the number of protein molecules that can form a monolayer around each AgNP. ${ }^{10}$ A simple calculation for the $15 \mathrm{~nm} \mathrm{NP}$ gives values of 43, 70, and 150 for the maximum number of HSA, TTR, and LYS protein molecules interacting with AgNP. These values are close to the ratio used in this experiment, indicating that the ratio of free protein to protein interacting with AgNP is not too far from 1:1 assuming an average coverage of NP of $50 \%$. These measurements were possible due to the highly collimated and small cross section synchrotron light beam of the B23 beamline, which is a unique feature among the current operational synchrotron beamlines dedicated to SRCD measurements.

The different behavior of LYS with AgNP can be rationalized based on its high isoelectric point of around 9.5; in the experimental conditions at $\mathrm{pH} 7.0$, lysozyme is positively charged thus greatly increasing its electrostatic interaction with the negatively charged AgNP, as compared to the other two proteins. This is confirmed by calculating the electrostatic surface potentials for the three proteins at $\mathrm{pH} 7.0$ and mapping them onto the threedimensional structure. Figure 3 shows that both HSA and hTTR have an overall negative charge with some limited positive patches while LYS is almost completely positive thus greatly enhancing its potential interaction with the negatively charged AgNP.

The thermal stability of proteins can be assessed by CD by following changes in the spectra with increasing temperature. To measure the relative stability of HSA in the presence of gold and silver nanoparticles, we have collected the whole CD spectra from 190 to $260 \mathrm{~nm}$ in the $20-90^{\circ} \mathrm{C}$ temperature range in steps of $2{ }^{\circ} \mathrm{C}$. This set of 35 spectra has been then analyzed by singular value decomposition to get information about the number of states contributing to the whole data set. ${ }^{11}$ In all three systems analyzed (HSA free, HSA-AuNP, and HSA-AgNP) the thermal unfolding can be described by using only two basis spectra: the "folded" starting spectrum containing the $\alpha$-helical structure and the "unfolded" spectrum containing some residual irregular structure (see Supporting Information, Figure S3). This indicates that in all cases the unfolding process can be described without the presence of an intermediate state. ${ }^{12}$ The change in the fraction of the "folded" spectrum obtained by singular value decomposition as a function of temperature has been used to estimate the relative stability of the HSA protein in the three different systems. Figure 4 shows the thermal unfolding of HSA $5 \mu \mathrm{g} / \mathrm{mL}$ (corresponding to a molar concentration of $76 \mathrm{nM}$ ), HSAAgNP, and HSA-AuNP.

The decreasing of the folded state with temperature has been fitted with a sigmoid curve for a simple two-state unfolding process; the resulting melting temperature and the rate constant of the unfolding process are reported in Table S1 (Supporting Information).

The thermal unfolding of free HSA is irreversible, has a melting temperature $\left(T_{M}\right)$ of $75.1{ }^{\circ} \mathrm{C}$, and shows a steep unfolding transition with a width of 6.3 , indicative of a highly cooperative unfolding reaction. These results are in good agreement with reported values of $76^{\circ} \mathrm{C}$ for the thermal unfolding of free HSA at $50 \mu \mathrm{g} / \mathrm{mL}$ concentration (corresponding to a molar concentration of $760 \mathrm{nM}) .{ }^{13}$ The thermal unfolding of HSA in the HSA-AuNP 
system shows a very similar highly cooperative unfolding reaction with a $T_{M}$ of $74.8^{\circ} \mathrm{C}$. In contrast, the unfolding of HSA in the presence of AgNP is less cooperative and has a significantly lower melting temperature of $69.1^{\circ} \mathrm{C}$. The highly cooperative unfolding reactions of $\mathrm{HSA}$ and $\mathrm{HSA}-\mathrm{AuNP}$ indicate that the protein existed initially as a compact, well-folded structure, while the gradual, less cooperative unfolding of HSA-AgNP suggests that the protein existed initially as a more flexible, partially unfolded protein or as a heterogeneous population of folded structures. Unfortunately, due to the nonreversibility of the thermal unfolding process, it is not possible to obtain true thermodynamic parameters, but it is possible to establish the relative stability of HSA interacting with nanoparticles. The comparison of the unfolding of HSA in the three conditions (free, $\mathrm{Ag}-, \mathrm{Au}-$ ) indicates that upon interaction with AuNP the protein does not alter its overall secondary structure or its stability. Upon interaction with AgNP the protein reduces its thermal stability with a decrease of $6{ }^{\circ} \mathrm{C}$ in the melting temperature and seems to exist initially with a more flexible folded structure, even if the destabilization due to the interaction with silver nanoparticles is not evident from the CD spectrum alone.

In summary we have shown that with synchrotron radiation circular dichroism it is possible to analyze the secondary structure of proteins in the low nanomolar concentration range, thus providing a unique method for detecting the relative stability of key biological proteins interacting with nanoparticles. This extreme sensitivity has allowed us to show that human serum albumin is significantly destabilized when interacting with silver nanoparticles, while its stability is not affected when interacting with gold nanoparticles. The high sensitive of this technique provides structural information on protein-NP complexes at near equimolar ratios and allows accessing detailed information that has been very difficult to obtain until now.

Methods. The gold nanoparticles were made by first heating $100 \mathrm{~mL}$ of a $2.5 \mathrm{mM}$ sodium citrate solution to $96^{\circ} \mathrm{C}$ in a microwave reactor (Discover $S$ by CEM). After $5 \mathrm{~min}$ was allowed for temperature stabilization, $5 \mathrm{~mL}$ of a $10 \mathrm{mM}$ solution of $\mathrm{HAuCl}_{4}$ was injected with vigorous stirring into the sodium citrate solution and the reaction allowed to go to completion by continued heating at $96{ }^{\circ} \mathrm{C}$ for $20 \mathrm{~min}$.

The silver nanoparticles were made by first heating $100 \mathrm{~mL}$ of a $2.5 \mathrm{mM}$ sodium citrate solution to $96{ }^{\circ} \mathrm{C}$ in a microwave reactor. After $5 \mathrm{~min}$ was allowed for temperature stabilization, $0.5 \mathrm{~mL}$ of $0.1 \mathrm{M} \mathrm{AgNO}_{3}$ solution was added and allowed to stabilize for $1 \mathrm{~min}$ before adding $1 \mathrm{~mL}$ of a $0.1 \mathrm{M}$ solution of ice cold $\mathrm{NaBH}_{4}$. On addition of the reducing agent, the initially colorless solution becomes first dark brown before changing to a clear transparent yellow solution.

Both AuNP and AgNP have been dialyzed against a $1 \mathrm{mM}$ phosphate buffer, $\mathrm{pH} 7.0$, prior to $\mathrm{CD}$ experiments. The dynamic light scattering data have been measured with a Malvern zeta sizer; measurements of free, dialyzed, AuNP, and AgNP show monodispersed samples with average hydrodynamic radii of 16.8 and $15.8 \mathrm{~nm}$, respectively.

Human serum protein (essentially fatty acid and globulin free) and chicken egg lysozyme were purchased from Sigma; recombinant human transtyrethrin was a kind gift from Professor Mizuguchi. Protein samples were prepared by dissolving them in $1 \mathrm{mM}$ phosphate buffer at $\mathrm{pH}$ 7.0. SRCD spectra were collected at the beamline B23 module end station B of Diamond Light Source (U.K.). SRCD spectra were collected with Suprasil cells of 1 and/or 10 path length (Starna and Hellma), with bandwidth of $1.2 \mathrm{~nm}$, integration time of $1 \mathrm{~s}, 1 \mathrm{~nm}$ digital resolution, $39 \mathrm{~nm} /$ min scan speed, and four repeated scans per spectrum. Reported spectra have been averaged, corrected by subtraction of CD spectrum of the buffer alone and smoothed by applying a sevenpoint Savitzky-Golay smoothing function.

Thermal unfolding had been monitored using $0.005 \mathrm{mg} / \mathrm{mL}$ HSA protein samples with a Peltier temperature controlled $1 \mathrm{~cm}$ Suprasil cell. CD spectra were recorded every $2{ }^{\circ} \mathrm{C}$ in the $20-90^{\circ} \mathrm{C}$ temperature range. Temperature was raised by $1{ }^{\circ} \mathrm{C}$ per minute and was allowed to equilibrate for $2 \mathrm{~min}$ before collecting the spectrum. The thermal unfolding data has been fitted to a Boltzmann equation of the type $A+(B-A) /(1+$ $\exp \left(x-x_{0}\right) / \mathrm{d} x$ ) (where $x$ is the temperature, $x_{0}$ is the melting temperature, and $\mathrm{d} x$ the width of the thermal transition) using a nonlinear square fitting algorithm with the software program Origin (OriginLab).

The changes in secondary structure of hTTR upon interaction with AgNP have been estimated by using the basis sets for the different secondary structure elements in the $200-240 \mathrm{~nm}$ region in the program $\mathrm{K} 2 \mathrm{D} 2 .^{14}$

The three-dimensional structures of HSA, hTTR, and LYS proteins have been derived from the protein data bank (access codes 1BM0, 1F41, 2YVB) and visualized using the PyMol program. Electrostatic surface potentials at $\mathrm{pH} 7.0$ have been calculated using the APBS program, the PropKa program has been used to assign the correct protonation states at $\mathrm{pH} 7.0$, and the PDB2PQR server used to convert input files from the $\mathrm{pdb}$ format to the pqr format. ${ }^{15-17}$

\section{ASSOCIATED CONTENT}

S Supporting Information. Additional information with SRCD spectra of HSA (Figures S1, S2, and S3) and thermal unfolding parameters (Table S1). This material is available free of charge via the Internet at http://pubs.acs.org.

\section{AUTHOR INFORMATION}

\section{Corresponding Author}

*luigi.calzolai@jrc.ec.europa.eu.

\section{ACKNOWLEDGMENT}

We would like to thank Prof. Mizuguchi (University of Toyama, Japan) for the kind gift of recombinant hTTR, and Diamond Light Source for beamtime award to B23 beamline for project SM-6192. The research leading to these results has received funding from the European Community's Seventh Framework Programme (FP7/ 2007-2013) under grant agreement $n^{\circ} 226716$.

\section{REFERENCES}

(1) Nel, A. E.; Madler, L.; Velegol, D.; Xia, T.; Hoek, E. M.; Somasundaran, P.; Klaessig, F.; Castranova, V.; Thompson, M. Nat. Mater. 2009, 8 (7), 543-57.

(2) Lundqvist, M.; Stigler, J.; Elia, G.; Lynch, I.; Cedervall, T.; Dawson, K. A. Proc. Natl. Acad. Sci. U.S.A. 2008, 105 (38), 14265-70.

(3) Deng, Z. J.; Liang, M.; Monteiro, M.; Toth, I.; Minchin, R. F. Nat. Nanotechnol. 2010, 6 (1), 39-44.

(4) Calzolai, L.; Franchini, F.; Gilliland, D.; Rossi, F. Nano Lett. 2010, 10 (8), 3101-5.

(5) Fasman, G. D., Circular dichroism and the conformational analysis of biomolecules; Plenum Press: New York, 1996.

(6) Greenfield, N. J. Nat. Protoc. 2006, 1 (6), 2876-90. 
(7) Shang, W.; Nuffer, J. H.; Muniz-Papandrea, V. A.; Colon, W.; Siegel, R. W.; Dordick, J. S. Small 2009, 5 (4), 470-6.

(8) Javorfi, T.; Hussain, R.; Myatt, D.; Siligardi, G. Chirality 2010, 22 (Suppl 1), E149-53.

(9) Wallace, B. A. Q. Rev. Biophys. 2009, 42 (4), 317-70.

(10) Mattoussi, H.; Mauro, J. M.; Goldman, E. R.; Anderson, G. P.; Sundar, V. C.; Mikulec, F. V.; Bawendi, M. G. J.Am. Chem. Soc. 2000, 122 (49), 12142-12150.

(11) Konno, T. Protein Sci. 1998, 7 (4), 975-82.

(12) Greenfield, N. J. Nat. Protoc. 2006, 1 (6), 2527-35.

(13) Wetzel, R.; Becker, M.; Behlke, J.; Billwitz, H.; Bohm, S.; Ebert, B.; Hamann, H.; Krumbiegel, J.; Lassmann, G. Eur. J. Biochem. 1980, 104 (2), 469-78.

(14) Perez-Iratxeta, C.; Andrade-Navarro, M. BMC Struct. Biol. 2008, 8 (1), 25.

(15) Li, H.; Robertson, A. D.; Jensen, J. H. Proteins 2005, 61 (4), 704-21.

(16) Baker, N. A.; Sept, D.; Joseph, S.; Holst, M. J.; McCammon, J. A. Proc. Natl. Acad. Sci. U.S.A. 2001, 98 (18), 10037-41.

(17) Dolinsky, T. J.; Nielsen, J. E.; McCammon, J. A.; Baker, N. A. Nucleic Acids Res. 2004, 32 (Web Server issue), W665-W667. 